\title{
Endovascular Treatment of Very Small Intracranial Aneurysms
}

\author{
A. ISKANDAR, J. NEPPER-RASMUSSEN \\ Department of Radiology, Odense University Hospital; Odense C, Denmark
}

Key words: intracranial aneurysm, endovascular treatment, complication, outcome, subarachnoid hemorrhage, intraprocedural rupture

\section{Summary}

The endovascular treatment of intracranial aneurysms $3 \mathrm{~mm}$ or less is considered controversial. The purpose of this study is to report angiographic and clinical results following coiling of such aneurysms and compare them to those of larger aneurysms (> $3 \mathrm{~mm})$.

Between November 1999 and November 2009 endovascular treatment was attempted in 956 consecutive intracranial aneurysms. Of 956 aneurysms, 111 aneurysms were very small aneurysms with a maximal diameter of $3 \mathrm{~mm}$ or less. We conducted a retrospective analysis of angiographic and clinical outcome following coiling of very small aneurysms and subsequently comparing it to the results of larger aneurysms.

Coiling initially failed in eight aneurysms. In the remaining 103 aneurysms endovascular treatment was accomplished and immediate angiographic results showed complete aneurysm occlusion in 43 aneurysms, nearly complete aneurysm occlusion in 54 aneurysms and less than 90\% aneurysm occlusion in six aneurysms. Complications occurred in the treatment of 15 aneurysms, including eight procedural ruptures, six thromboembolic events and one case of early hemorrhage. Compared with larger aneurysms, treatment of very small aneurysms was associated with a higher rate of procedural ruptures $(7.2 \%$ versus $4.4 \%$ ) and procedural mortality (4.7\% versus $2.7 \%$ ) but a lower procedural morbidity $(1.9 \%$ versus $4.0 \%)$. However none of these differences reached statistical significance $(p=0.186$, $p=0.388$, respectively). The retreatment rate was higher for the larger aneurysms $(8.2 \%$ and $6.3 \%)$, but this was not significant either $(p=0.496)$. At nine-month follow-up significantly more small aneurysms were found to have a stable occlusion grade compared to large aneurysms.

Endovascular treatment of very small aneu- rysms is feasible with a lower retreatment rate compared to large aneurysms (>3 mm). However the data also suggest that endovascular treatment of very small aneurysms might be associated with an increased risk of procedural ruptures and mortality. At nine-month follow-up results indicate significantly less compaction in the very small aneurysms.

\section{Introduction}

The International Subarachnoid Aneurysm Trial (ISAT) documented that coiling produces better patient outcomes compared to surgery for those who are equally suited for both endovascular coil treatment and surgical clipping 1. However the number of very small aneurysms $(\leq 3 \mathrm{~mm})$ that were included in this study was limited and the endovascular treatment of these aneurysms remains controversial. Few published studies focus on the outcome of endovascular treatment for very small aneurysms 2-7. The aim of this study is to evaluate in our institution the angiographic and clinical outcome of endovascular treatment of intracranial aneurysms $\leq 3 \mathrm{~mm}$ and compare these results with those of larger aneurysms $(>3 \mathrm{~mm})$.

\section{Materials and Method}

\section{Patients}

This is a retrospective study of a prospectively maintained database. From November 1999 to November 2009, 956 consecutive intracranial aneurysms in 934 patients underwent endovascular treatment at Odense University Hospital which functions as a tertiary referral center. During this time frame data from these patients 
were recorded into a database. In the same period 189 patients underwent surgical clipping at the same institution. All patients were evaluated in a conference between a neurosurgeon and an interventional neuroradiologist. In general, endovascular treatment was the preferred treatment unless it was thought not to be possible in the vast majority of the cases because of one of the following reasons: 1) severe vasospasm, 2) severe atherosclerosis, 3) unfavorable dome-to-neck ratio (typically dome-to-neck ratio $<1), 4$ ) essential arteries arising from the aneurysm wall and 5) not possible to obtain a useful working projection to display the aneurysm neck. In seven cases 'patients' were in a very critical clinical state and they had to be taken directly to surgery without preceding angiography. Eight patients were randomized to surgical clipping as part of the ISAT study. Mycotic aneurysms were excluded from the study since these aneurysms are not standardly treated with coils at our institution. Except for the mycotic aneurysms no other aneurysms were excluded unless endovascular treatment was thought not to be possible because of one of the above mentioned reasons. Hence dissecting aneurysms as well as large and giant aneurysms were included in the study. Aneurysm size was defined as the maximal aneurysm diameter. Of the 956 aneurysms, 111 aneurysms in 107 patients had a maximal diameter of $3 \mathrm{~mm}$ or less. Table 1 shows pertinent patient demographic information and aneurysm location.

\section{Hunt and Hess Grading}

Of the 111 very small aneurysms, 87 were ruptured and 24 were not. Of these 24 aneurysms, four aneurysms in four patients were treated because of patient preference, three aneurysms in three patients had a strong family history of rupture and 17 aneurysms in 17 patients were in addition to another ruptured aneurysm. The pretreatment clinical condition of all the patients except for the seven patients with solely unruptured aneuryusms was graded according to the Hunt and Hess grade $(\mathrm{HH})^{8}$. Six patients were $\mathrm{HH} 0,57$ patients were $\mathrm{HH}$ I to II, 15 patients were $\mathrm{HH}$ III and 22 patients were $\mathrm{HH}$ IV to V.

\section{Timing of Treatment}

As a general rule at the institution, the timing of treatment after subarachnoid hemorrhage is within 48 hours. In 14 of the 87 rup-

Table 1 Characteristics of patients and aneurysms in the study.

\begin{tabular}{|l|l|l|l|}
\hline & $\begin{array}{l}\text { Very small aneurysms } \\
(\mathbf{3} \mathbf{~ m m ~})\end{array}$ & $\begin{array}{l}\text { Large aneurysms } \\
\mathbf{( > 3} \mathbf{~ m m})\end{array}$ & P-value \\
\hline No. of patients & 107 & 827 & \\
\hline No. of aneurysms & 111 & 845 & 0.763 \\
\hline Female (\%) & $73(68.2)$ & $576(69.6)$ & 0.424 \\
\hline Mean age & 50.7 & 53.1 & 0.062 \\
\hline Ruptured aneurysms (\%) & $87(78.4)$ & $720(85.2)$ & 0.333 \\
\hline Aneurysms location (\%) & & & 0.712 \\
$\quad \begin{array}{l}\text { Anterior cerebral artery } \\
\text { Middle cerebral artery } \\
\text { Internal carotid artery }\end{array}$ & $50(45)$ & $340(40)$ & 0.010 \\
Posterior circulation & $12(11)$ & $82(10)$ & $242(29)$ \\
\hline
\end{tabular}

Table 2 Dome to neck ratio for the very small aneurysms $(\leq \mathbf{3} \mathbf{~ m m})$.

\begin{tabular}{|l|l|}
\hline Dome to neck ratio $(\mathbf{x})$ & Number of aneurysms \\
\hline$X \leq 1$ & 33 \\
\hline $1<\mathrm{x} \leq 1.5$ & 67 \\
\hline $1.5<\mathrm{X} \leq 2.0$ & 7 \\
\hline$X>2.0$ & 4 \\
\hline
\end{tabular}


tured aneurysms, treatment was delayed beyond 15 days after rupture. The delays were due to the following reasons: Aneurysm was not detected on initial angiography in six patients, failed surgery at other hospitals in five patients, one patient had a warning leak recognized later, one patient was clinically unstable and one patient had a recanalization after primary treatment at another hospital.

\section{Angiographic Technique}

All patients underwent conventional angiography of both carotid arteries and vertebral arteries. The patients were treated under general anesthesia with systemic heparinization. 3000$5000 \mathrm{U}$ of intravenous heparin were given in both ruptured and non-ruptured aneurysms followed by heparinized catheter flush. The heparin treatment was continued 24 hours after the procedure. If stent was used, clopidogrel $(75 \mathrm{mg}$ ) and aspirin $(150 \mathrm{mg})$ were administered for two and six months, respectively.

The assessment of the aneurysms as well as the embolization was performed on Philips Integris BV5000 with 3D angiography in all patients. In most cases, the diagnostic cerebral angiogram and embolization of the ruptured aneurysm was performed in the same procedure.

Five interventional neuroradiologists were involved in the coiling of the aneurysms, and they all had more than one year of experience with endovascular coil embolization prior to treatment of the investigated aneurysms.

In the majority of cases, the aneurysms were occluded with 2 or $3 \mathrm{~mm}$ coils, mainly Guglielmi detachable coils (Boston Scientific, Fremont, CA, USA), Trufill orbit detachable coils (Cordis, Miami, FL, USA), Micrus coils: (Micrus endovascular, San Jose, CA, USA) and Axium coils (ev3 Inc., Plymouth, MN, USA).

Balloons and stents were used when it was thought that they would be useful, typically in aneurysms with a wide neck. The dome/neck ratio of the small aneurysms is seen in Table 2.

In case of a procedural rupture, protamine was administered and a continued attempt to close the aneurysm with coils was made. If a treatment was abandoned, it was recorded along with the reason. The procedural complications were registered. Based on the neurosurgical medical records all delayed complications as well as clinical outcome at the time of discharge were registered in all patients. If mortality or deficits were thought to be related to the endovascular treatment, this was noted accordingly. Any mortality and deficits following a procedural rupture was considered to be caused by the rupture.

\section{Angiographic Results and Follow-up}

Immediate angiographic results of coiling were classified into three outcomes by the RoyRaymond Classification 9: 1) complete occlusion (defined as no residual filling of the aneurysm sac or neck), 2) near complete (defined as no filling of the sac but inflow in coil at the neck or a present neck remnant) and 3) incomplete occlusion (defined as a persistent filling of the sac). Patients were scheduled for a follow-up visit nine months after the procedure. The follow-up visit was based on both physical examination and imaging studies, including both conventional angiography and magnetic resonance angiography. However in patients over 70 years, only magnetic resonance angiography was done during the follow-up. Clinical outcome was classified according to the Glasgow Outcome Scale (GOS). The imaging results were classified into the same three outcomes as the initial angiographies and it was noted whether the occlusion state was increased, decreased or unchanged. If the result was complete occlusion, no additional imaging follow-up was made. If there were no discrepancy between conventional angiography and magnetic resonance angiography, only magnetic resonance angiography was done if further follow-up was needed.

\section{Statistical Analysis}

The Chi- square test was used for comparison of frequencies incorporating Yates' correction for continuity when appropriate. Level of significance was set at $\mathrm{p}$-value $<0.05$.

\section{Results}

\section{Initial Treatment Results}

Endovascular treatment was attempted in 111 very small aneurysms in 107 patients. Thirteen aneurysms were treated with balloon assistance (10 Hyperform (ev3 Inc, Plymouth, MN, USA), two Hyperglide (ev3 Inc, Plymouth, MN, USA) and one Sentry (Boston Scientific, Fremont, CA, USA)) and stent placement was 
used in the treatment of eight aneurysms (1 LEO (BALT Co., Montmorency, France), three Enterprise (Cordis, Miami, FL, USA) and four Neuroform (Boston Scientific, Fremont, CA, USA). The treatment failed in eight aneurysms, which are included in the analysis. In four cases the neck was too wide to retain the coils and in four cases the operator was unable to catheterize the aneurysms. Four of these eight aneurysms were subsequently successfully clipped. One patient was retreated with balloon assistance with an initial good result, but two hours after the procedure the patient developed intracranial bleeding from a vessel not related to the coiled aneurysm and died. This patient is also listed below as a case of procedure-related mortality. One patient was once stable transferred back to the original hospital, and we have no further information on this patient. Finally in two cases the parent artery was sacrificed in a later procedure. Immediate angiographic results are shown in Table 3 .

\section{Procedure-Related Complications}

Procedural complications occurred in $15 \mathrm{pa}$ tients $(14.0 \%)$. Complications included eight cases of procedural rupture, six cases of thrombombolic events and one case also mentioned in paragraph above, who during retreatment developed early (within 24 hours) hemorrhage from a vessel not related to the treated aneurysm. Five patients died ( $4.7 \%$, four procedural ruptures and one early hemorrhage) and two patients had morbidity $(1.9 \%$, both thromboembolic events). In eight cases (four procedural ruptures and four thromboembolic events) no procedure caused mortality or persistent morbidity. All complications occurred in ruptured aneurysms except one event of thromboembolism and the event of early hemorrhage, which both happened in unruptured aneurysms.

\section{Recurrent Hemorrhage}

One patient developed a new aneurysm eight years after initial embolization, and four weeks after successful embolization of the second aneurysm, she had a new bleed from the latest treated aneurysm. The patient underwent endovascular retreatment of the last mentioned aneurysm, however she developed persistent neurological deficits (modified Rankin Scale score of 3 ).

\section{Nine-Month Follow-up}

Angiography and clinical follow-up at nine months after first treatment was performed in 73 aneurysms $(65.8 \%)$ of the 111 aneurysms. In 36 patients with 36 aneurysms, the follow-up data are not available for the following reasons: ten patients had died, six patients were treated with occluded parent artery or clipping, two patients refused follow-up, four patients were dependent at nursing homes, four are followed in other hospitals, ten patients were recently treated and therefore their nine-month follow-up is scheduled in the future. Of the 73 aneurysms, 56 $(76,7 \%)$ were complete occluded and further follow-up of these patients was not needed. Fourteen aneurysms (19.2\%) were nearly complete occluded. Four of these 14 aneurysms did not need further follow-up because the occlusion grade was unchanged. In the remaining ten aneurysms, the occlusion was decreased compared to the initial results, and they were scheduled for further follow-up, at which because of unchanged or increased occlusion they did not need further follow-up. Of the 73 aneurysms, three aneurysms $(4.1 \%)$ were incompletely occluded. Additional coiling was attempted in two of these aneurysms and was successful in both cases. Both of these aneurysms had reopened by coil compaction, and continued follow-up was not deemed necessary at first follow-up after additional treatment. The third incompletely oc-

Table 3 Immediate angiographic results following endovascular treatment of very small aneurysms.

\begin{tabular}{|l|l|l|}
\hline Initial treatment results & $\begin{array}{l}\text { Unruptured aneurysms } \\
\mathbf{8 7} \text { aneurysms }\end{array}$ & $\begin{array}{l}\text { Ruptured aneurysms } \\
\mathbf{2 4} \text { aneurysms }\end{array}$ \\
\hline Complete occlusion (\%) & $32(36.8)$ & $11(45.8)$ \\
\hline Nearly complete occlusion (\%) & $43(49.4)$ & $11(45.8)$ \\
\hline Less than 90\% occlusion (\%) & $6(6.9)$ & $0(0)$ \\
\hline Abandoned treatment (\%) & $6(6.9)$ & $2(8.3)$ \\
\hline
\end{tabular}


cluded aneurysm was not offered additional treatment, because the occlusion was judged to be stable and further treatment was not possible. Table 3 shows the outcome at nine-month follow-up for both small and larger aneurysms; significantly fewer very small aneurysms needed further follow-up at that point.

\section{Retreatment}

Seven of the 111 aneurysms underwent retreatment. One aneurysm was retreated with coiling after initial failed endovascular treatment, four aneurysms were retreated with clipping shortly after failed endovascular treatment, and two aneurysms were retreated with coiling at the nine-month follow-up.

\section{Comparison of Very Small Aneurysms with Larger Aneurysms.}

Table 4 summarizes the results from endovascular treatment of the very small aneurysms and compare these results with those of aneurysms larger than $3 \mathrm{~mm}$.

\section{Discussion}

This study suggests that endovascular treatment of very small aneurysms $(\leq 3 \mathrm{~mm})$ is technically feasible and demonstrates better rates of complete or nearly complete occlusion at follow-up resulting in significantly lower retreat- ment rates as compared to larger aneurysms (> $3 \mathrm{~mm}$ ). Furthermore procedural morbidity was found to be lower in very small aneurysms. However the data suggest that small aneurysms size $(\leq 3 \mathrm{~mm})$ is a risk factor for procedural ruptures and subsequent mortality, although these results did not meet statistical significance. Very small aneurysms $(\leq 3 \mathrm{~mm})$ constituted $11.6 \%$ of all aneurysms treated with endovascular treatment at our institution. The result from the endovascular treatment of these very small aneurysms was compared with the results from the larger aneurysms. The occurrence of ruptured aneurysms was similar in the two groups. Furthermore the location of the aneurysms was comparable for both groups except that larger aneurysms $(>3 \mathrm{~mm})$ were significantly more common on the carotid artery. The vast majority of the very small aneurysms had a dome-toneck ratio of $\leq 1.5$. Procedural complications for the very small aneurysms occurred with an overall prevalence of $14.0 \%$, however intra-procedural complications leading to either mortality or morbidity occurred at a rate of $6.5 \%$, the rest of the complications remained without clinical sequelae. Procedure-related ruptures are one of the most significant complications of coiling. In several previous published studies very small aneurysms has been found to be associated with a higher risk of rupture ${ }^{4,7,10}$. This is confirmed by our study. In our study intraprocedural ruptures occurred at a higher rate in very small aneurysms compared to larger aneurysms $(7.2 \%$ versus $4.4 \%)$, although this differ-

Table 4 Comparison of results of very small aneurysms and larger aneurysms.

\begin{tabular}{|c|c|c|c|}
\hline & $\begin{array}{l}\text { Very small aneurysms } \\
(\leq \mathbf{3} \mathbf{~ m m})\end{array}$ & $\begin{array}{l}\text { Large aneurysms } \\
(>\mathbf{3} \mathrm{mm})\end{array}$ & P-value \\
\hline Procedural mortality (\%) & $5(4.7)$ & $22(2.7)$ & 0.388 \\
\hline Procedural morbidity (\%) & $2(1.9)$ & $33(4.0)$ & 0.381 \\
\hline Procedural ruptures (\%) & $8(7.2)$ & $37(4.4)$ & 0.186 \\
\hline $\begin{array}{l}\text { Morbidity/mortality following } \\
\text { procedural ruptures (\%) }\end{array}$ & $4(3.7)$ & $18(2.2)$ & 0.507 \\
\hline Treatment abandoned (\%) & $8(7.2)$ & $34(4.0)$ & 0.196 \\
\hline $\begin{array}{l}\text { Retreatment rate }(\%) \\
\text { Coiling }(\%) \\
\text { Clipping }(\%)\end{array}$ & $\begin{array}{l}7(6.3) \\
3(2.7) \\
4(3.6)\end{array}$ & $\begin{array}{l}69(8.2) \\
53(6.3) \\
16(1.9)\end{array}$ & $\begin{array}{l}0.496 \\
0.132 \\
0.406\end{array}$ \\
\hline $\begin{array}{c}\text { Adjunctive devices (\%) } \\
\text { Balloon (\%) } \\
\text { Stent }(\%)\end{array}$ & $\begin{array}{l}21(18.9) \\
13(11.7) \\
8(7.2)\end{array}$ & $\begin{array}{l}167(19.8) \\
113(13.4) \\
54(6.4)\end{array}$ & $\begin{array}{l}0.834 \\
0.626 \\
0.742\end{array}$ \\
\hline $\begin{array}{l}\text { No further follow up/treatment } \\
\text { needed 9-month follow-up (\%) }\end{array}$ & $61(83.6)$ & $414(71.3)$ & 0.026 \\
\hline
\end{tabular}


ence was not significant $(p=0.186)$. Sluzewski et al. ${ }^{10}$ found that intraprocedural ruptures results in one of two extremes, either the patient recovers well or the patient die. This trend is also seen in our study; half of the patients with a procedure-related rupture died while the other half survived without morbidity. The rates of procedural ruptures found in our study are in the same range as the published results 2,7 .

Van Rooij et al. ${ }^{7}$ found that although the endovascular treatment of very small aneurysms $(\leq 3 \mathrm{~mm})$ was associated with a more twofold increase in the incidence of procedural ruptures compared with larger aneurysms, this did not result in an increased mortality or morbidity. Our study results however propose that there might be a higher procedural mortality associated with endovascular treatment of small aneurysms as compared to larger aneurysms. When very small aneurysms rupture during endovascular treatment, the whole aneurysm is often blown away. In our experience it is technically more challenging to continue the procedure and obtain occlusion of the aneurysm in this situation. Fatal outcome of the endovascular treatment mainly happened in patients in whom a procedural ruptures had occurred (four out of the five patients who died had a procedural rupture). The increased rate of mortality associated with endovascular treatment of the small aneurysms is most likely caused by the increased risk of procedural ruptures as reported in previous publications ${ }^{2,4}$. Mortality is therefore expected to be reduced with the development of techniques to prevent ruptures as well as control hemorrhage in the event of rupture. The technical difficulties in the management of very small aneurysms has prompted proposal of alternative techniques ${ }^{4,11-14}$.

Based on their meta-analysis Brinjikji et al. ${ }^{2}$ reported a retreatment rate of $5.4 \%$ for aneurysms $\leq 3 \mathrm{~mm}$. In the study by Van Rooij et al. ${ }^{7}$ the retreatment rate for these very small aneurysms was $4.6 \%$, which was found to be significantly lower than for larger aneurysms $(10.0 \%)$. Our results confirm this finding in that we find a lower retreatment rate in small aneurysms compared to larger aneurysms $(6.3 \%$ versus $8.2 \%$ ), although this result was not significant $(\mathrm{p}=0.496)$. The majority of the aneurysms (76.7\%) were completely occluded at the ninemonth follow-up. Only three aneurysms were found to be incompletely occluded and retreat- ment with coiling was attempted and succeeded in two aneurysms. These rates are comparable with those reported in the literature ${ }^{3,15}$. Evaluation of the occlusion grade at nine-month follow-up showed that significantly more very small aneurysms did not need further evaluation and follow up was deemed not necessary. This suggests that when a small aneurysm is treated with coiling, there is less risk of compaction compared to large aneurysms.

This study has several limitations. It is single center retrospective study. During the time frame of the study more sophisticated endovascular materials were available. With the development of softer, smaller coils, more flexible microcatheters and microwires as well as more refined adjunctive techniques, the endovascular treatment of very small aneurysms and therefore the treatment results from the initial years included in this study is less likely to reflect the current endovascular treatment outcomes. In addition, as noted by Nguyen et al. ${ }^{4}$ the use of the maximal aneurysm diameter may not be the best estimate of the real risks of intra-procedural rupture related to size, because as they highlight: "some $5 \times 2 \mathrm{~mm}$ aneurysms may be at higher risk of perforation than certain round $4 \mathrm{~mm}$ aneurysms". As with other previously published data, the power of this study is limited owing to a small number of events, and the results can therefore not be viewed as definitive. More data are required to further clarify the risk and benefits associated with endovascular treatment of very small aneurysms. In addition a study comparing clipping and coiling of very small aneurysms may be needed to better decide on the optimal treatment (treatment of choice) of these aneurysms.

\section{Conclusion}

This study suggests that endovascular treatment of very small aneurysms $(\leq 3 \mathrm{~mm})$ is technically feasible with lower retreatment rate as compared to larger aneurysms. At nine-month follow-up results indicate significantly less compaction in the very small aneurysms. However the data also suggest that endovascular treatment of very small aneurysms might be associated with increased risk of procedural ruptures and mortality. 


\section{References}

1 Molyneux A, Kerr R, Stratton I, et al. Internationa Subarachnoid Aneurysm Trial (ISAT) of neurosurgical clipping versus endovascular coiling in 2143 patients with ruptured intracranial aneurysms: a randomised trial. Lancet. 2002; 360: 1267-1274.

2 Brinjikji W, Lanzino G, Cloft HJ, et al. Endovascular treatment of very small (3 $\mathrm{mm}$ or smaller) intracranial aneurysms: report of a consecutive series and a metaanalysis. Stroke. 2010; 41: 116-121.

3 Ioannidis I, Lalloo S, Corkill R, et al. Endovascular treatment of very small intracranial aneurysms. J Neurosurg. 2010; 112: 551-556.

4 Nguyen TN, Raymond J, Guilbert F, et al. Association of endovascular therapy of very small ruptured aneurysms with higher rates of procedure-related rupture. J Neurosurg. 2008; 108: 1088-1092.

5 Pierot L, Barbe C, Spelle L, ATENA investigators. Endovascular treatment of very small unruptured aneurysms: rate of procedural complications, clinical outcome, and anatomical results. Stroke. 2010; 41: 2855 2859.

6 Suzuki S, Kurata A, Ohmomo T, et al. Endovascular surgery for very small ruptured intracranial aneurysms. Technical note. J Neurosurg. 2006; 105: 777-780.

7 Van Rooij WJ, Keeren GJ, Peluso JP, et al. Clinical and angiographic results of coiling of 196 very small $(<$ or $=$ $3 \mathrm{~mm}$ ) intracranial aneurysms. Am J Neuroradiol. 2009; 30: $835-839$.

8 Hunt WE, Kosnik EJ. Timing and perioperative care in intracranial aneurysm surgery. Clin Neurosurg. 1974; 21: 79-89.

9 Roy D, Milot G, Raymond J. Endovascular treatment of unruptured aneurysms. Stroke. 2001; 32: 1998-2004.

10 Sluzewski M, Bosch JA, van Rooij WJ, et al. Rupture of intracranial aneurysms during treatment with Guglielmi detachable coils: incidence, outcome, and risk factors. J Neurosurg. 2001; 94: 238-240.

11 Gupta V, Chugh M, Jha AN, et al. Coil embolization of very small ( $2 \mathrm{~mm}$ or smaller) berry aneurysms: feasibility and technical issues. Am J Neuroradiol. 2009; 30 (2): 308-314.
12 Gil A, Vega P, Murias E, et al. Balloon-assisted extrasaccular coil embolization technique for the treatment of very small cerebral aneurysms. J Neurosurg. 2010; 112: 585-588.

13 Chen Z, Feng H, Tang W, et al. Endovascular treatment of very small intracranial aneurysms. Surg Neurol. 2008; 70: 30-35.

14 Henkes H, Reinartz J, Preiss H, et al. Endovascular treatment of small intracranial aneurysms: three alternatives to coil occlusion. Minim Invasive Neurosurg. 2006; 49: 65-69.

15 Hai J, Deng DF, Chen ZQ, et al. Endovascular embolization of small ruptured intracranial aneurysms using a biplane angiographic system with three-dimensiona rotational digital subtraction angiography. J Clin Neurosci. 2009; 16: 1028-1033.
Aline Iskandar, MD

Odense Universitetshospital Sdr. Boulevard 29

5000 Odense C, Denmark

Tel.: +1(978) 6770103

E-mail: aline_iskandar@yahoo.com 